\title{
CAPITAL SOCIAL DE LA COMUNIDAD DE TIMAR, REGIÓN DE ARICA Y PARINACOTA, COMO RECURSO TERRITORIAL PARA LA ADAPTACIÓN ANTE PERTURBACIONES AMBIENTALES
}

\author{
SOCIAL CAPITAL OF THE COMMUNITY OF TIMAR (ARICA Y PARINACOTA \\ REGION) AS A TERRITORIAL RESOURCE FOR ADAPTATION TO \\ ENVIRONMENTAL DISTURBANCES
}

Alejandro Tapia Tosetti*, José F. López Cepeda* y Oliver Meseguer Ruiz*

Se realizó una investigación participativa en la localidad de Timar, zona rural de Pampa Alta en el desierto de Atacama de la Región de Arica y Parinacota, en el extremo norte de Chile, se consideró la vulnerabilidad ambiental que posee la localidad y las respuestas comunitarias que se enmarcan teóricamente en el concepto de capital social. Los resultados dan cuenta de que existe un alto capital social compuesto por las relaciones de confianza entre residentes rurales y migrados urbanos, que funcionan como un recurso territorial para la respuesta a perturbaciones del sistema socioambiental.

Palabras claves: Capital social, vulnerabilidad, geografía ambiental, norte de Chile.

A participatory investigation was carried out in the locality of Timar, the rural area of high pampa in the Atacama Desert of the Region of Arica and Parinacota in the extreme north of Chile, it is considered the environmental vulnerability that owns the locality and its community forms of answers, theoretically framed in the concept of social capital. The results show that there is a high social capital composed of the trust relationships between the rural residents and the urban migrants, who function as a territorial resource for the response to the disturbances of the socio-environmental system.

Key words: social capital, environmental vulnerability, environmental geography, Northern Chile.

\section{Introducción}

El extremo norte de Chile, parte del desierto de Atacama, constituye un espacio que presenta condiciones naturales caracterizadas por una intensa aridez, originada por los efectos que ejercen en este sector de Sudamérica el anticiclón del Pacífico Suroriental y la corriente de Humboldt, que entregan gran estabilidad atmosférica, inhibiendo la ocurrencia de precipitaciones en el borde occidental del continente; esta condición árida se acentúa por la presencia de la alta cordillera andina que actúa como barrera, impidiendo la penetración de masas de aire húmedas provenientes del oriente, creando el efecto de sombra pluviométrica en su vertiente de sotavento (Aceituno 1997; Sarricolea y Romero 2015).

No obstante el predominio de la aridez, la ocurrencia de lluvias en el macizo andino durante el período estival aporta recursos hídricos que son utilizados no solo en el sector de montaña, sino que, producto del escurrimiento superficial y subterráneo, se originan pequeños cursos intermitentes que abastecen áreas específicas en el desierto nortino y que son aprovechados mediante distintas actividades y formas de organización que los habitantes rurales de la región han desarrollado a lo largo de los años (Núñez, 1974). La permanente interrelación entre las comunidades y el medio árido ha originado el rasgo más destacado de las sociedades asentadas en el sector, su capacidad de adaptación y manejo del entorno.

Además de la aridez, destaca como rasgo del clima regional la alta variabilidad interanual que presentan las condiciones climáticas, entendiéndose como tal las variaciones en el estado medio del clima en todas las escalas temporales y espaciales, asî como la ocurrencia de fenómenos extremos (IPCC 2013), que se traducen en alteraciones atmosféricas que expresan la influencia de algunos eventos meteorológicos que inciden sobre el área, como son, entre otros, la presencia del Monzón Amazónico, la acción de la Alta de Bolivia, así como El NiñoOscilación del Sur (ENOS) (Romero et al. 2013; Mendonça 2013).

\footnotetext{
* Universidad de Tarapacá, Departamento de Ciencias Históricas y Geográficas, Arica, Chile. Correo electrónico atapia@uta.cl; jlopezc@uta.cl; omeseguer@academicos.uta.cl
} 
La manifestación permanente de esta variabilidad climática en la región trae consigo incertidumbres respecto de la aparición de irregularidades de carácter ambiental que alteran las prácticas productivas habituales de los pobladores en el área rural de la región del extremo norte de Chile. Entre los fenómenos que se presentan destacan perturbaciones ambientales como sequías prolongadas, eventuales ocurrencias de lluvias locales abundantes que causan inundaciones y aluviones, así como también alteraciones en las temperaturas que pueden afectar las actividades productivas o favorecer la aparición de ondas de frío (acompañadas de nevadas y fuertes vientos), (Romero et al. 2013; Sarricolea y Romero 2015; Meseguer-Ruiz et al. 2016).

En el contexto de cambio global del clima, los modelos aplicados para el área andina del sector norte de Chile predicen reducciones en la disponibilidad de agua por menores precipitaciones, mayores temperaturas, evaporación y aumento en la variabilidad en el comportamiento interanual, lo que acentuará la crisis de disponibilidad del recurso hídrico (Sarricolea y Romero 2015), situación que compromete la conservación de la naturaleza, el desarrollo de la minería, de la agricultura y de la urbanización, cuestión que no es exclusiva del norte chileno, ya que este contexto de cambio global también ha sido constatado en otras áreas en el mundo (Adger 2003, Padigala 2015).

\section{Capital social y la comunidad de Timar}

Ante las condiciones de variabilidad y cambio climático que se presentan en esta área, resulta de interés abordar los aspectos fundamentales de las variaciones en los sistemas ecológicos y sociales que tienen lugar en la región y que pueden abordarse desde los enfoques del capital social. En ese sentido, las comunidades del lugar que históricamente han acudido a este recurso comunitario, con la finalidad de gestionar el territorio ante la incertidumbre que generan las condiciones propias de la zona o región, constituyen un eficaz observatorio para, con el concurso de sus miembros, acceder a la comprensión de las prácticas de mitigación y de adaptación desarrolladas ante este tipo de situaciones, que se presentan mediadas por la concepción cultural de los miembros en las comunidades involucradas, (Adger et al. 2013).

Las relaciones del capital social han sido abordadas desde la óptica de la Geografía como un recurso del territorio, es decir, un conjunto de relaciones multiescalares y multidimensionales, físicas y simbólicas, establecidas entre los miembros de una colectividad y entre estos y los ecosistemas que conforman la naturaleza de un lugar que, a su vez, forman parte de sistemas regionales mayores (López et al. 2017). Los objetivos apuntan al desarrollo económico, y en una perspectiva más amplia, abordan la sustentabilidad de un lugar expresada en sus componentes económicos, sociales y ambientales (Foronda-Robles y Gallindo-Pérez de Azpillaga 2012).

En estudios de capital social realizados con anterioridad en una comunidad de pastores altiplánicos en la región de Arica y Parinacota se reconocieron tres niveles: el primero está constituido por las relaciones de confianza y apoyo mutuo que se constituyen en el núcleo familiar, el segundo por la reciprocidad y colaboración a nivel comunitario y el tercer nivel está conformado por los vínculos que se establecen entre los dos niveles anteriores con instituciones de carácter público y privado (López et al. 2017).

El capital social de una comunidad se sustenta en las personas que la componen, de allí que la reducción de sus miembros, debido al éxodo y movilidad de su población, tiende a afectar la retroalimentación de sus habilidades adaptativas (Arriagada, 2003). En el caso de la precordillera durante la década de 1960, se vio intensificado el proceso de migración y movilidad, al punto de que muchas localidades, como Timar, perdieron parte de su población, debido a la atracción económica que significó la implementación de políticas económicas especiales para la provincia de Arica, que generó el arribo de población rural al área litoral; así por ejemplo, para 1943 la localidad contaba con 43 personas y ya para 1989 la cantidad de habitantes en el poblado había disminuido a 29 (González, 1990).

El desplazamiento de poblaciones desde las áreas interiores andinas hacia sectores costeros, si bien implicó que dejaran el espacio correspondiente a la comunidad de origen, no significó un rompimiento de los lazos de unión con la comunidad misma, ni desvinculación radical respecto de esta, más bien produjo un cambio y adecuación en los mecanismos de adscripción debido a la nueva situación, de manera que la comunidad, en la actualidad, está conformada por los miembros de la comunidad de origen y por integrantes que residen fuera de ella, en especial en el sector costero de la región, ciudad y valles, estableciéndose diversos niveles de asociatividad que expresan una situación de 
translocalización de las relaciones sociales de la comunidad (Gundermann y Vergara 2009).

La relación entre movimientos poblacionales, capital social y adaptación ante las perturbaciones ambientales ha sido abordada desde dos perspectivas, una que visualiza la migración como forma de ajuste, como estrategia de supervivencia que busca reducir la presión de la población sobre los escasos recursos, facilitando con ello la reducción de riesgos y mejorando las posibilidades de supervivencia (Scheffran et al. 2012), cuando ello sucede, los migrantes establecen redes en el lugar de arribo para ir en sustento del territorio de origen. La otra perspectiva sostiene que el éxodo poblacional de un territorio erosiona, en definitiva, la capacidad de reacción a distintas perturbaciones o variaciones ambientales, porque, junto con la salida de personas se va también el conocimiento histórico acumulado de reacción a disrupciones ambientales del pasado, debilitándose así la herencia en el conocimiento local para gestionar un riesgo o afrontar un desastre (Adger et al. 2005).

La población rural del norte de Chile ha utilizado en sus prácticas ancestrales un conjunto de herramientas concebidas a lo largo del tiempo, que aún con cambios y modificaciones sufridas en el transcurso de este le han permitido desarrollarse como sociedad en un espacio difícil por sus condiciones de altitud y aridez, posibilitando una eficaz interrelación con el medio natural y favoreciendo su adaptación al mismo, manejando los recursos de acuerdo con sus requerimientos e interviniéndolos adecuadamente (Alvarado, 1970).

La continuidad de la práctica de movilidad o desplazamiento llevada a cabo por comuneros de los pequeños oasis del desierto, que les permitía hacer uso de la dotación de recursos ofrecido por el medio, desarrollar relaciones de reciprocidad entre pobladores andinos, así como mantener un activo intercambio de bienes, servicios y vínculos simbólicos permanentes entre el territorio de origen y el territorio regional en que se inserta, fue impactada por el abandono y migración que afectó a los espacios interiores de Arica.

Glaeser et al. 2002 señalan que las conexiones sociales, base del capital social estructural, caen notablemente con la distancia física y con los costes de viaje. Por tanto, es probable que los movimientos migratorios ocurridos en los últimos años hayan originado un impacto en el capital social que disponen las comunidades, socavando la capacidad de hacer frente a nuevas irrupciones que se presentan en el territorio afectando sus condiciones de vida, producto de la recurrencia de fenómenos naturales asociados al aumento de la variabilidad climática y al cambio global del clima.

Es posible preguntar si la movilidad de la población rural en los oasis-quebradas del extremo norte de Chile ha significado una pérdida de capital social adaptativo o, por el contrario, constituye un recurso al que la comunidad del lugar puede apelar ante una disrupción que altere su sistema socioecológico.

El presente trabajo tiene como objetivo reconocer la disponibilidad de capital social en la comunidad de Timar, y su rol como un recurso que permite y contribuye a enfrentar con éxito las perturbaciones que se manifiestan en el sistema socionatural que lo conforman. El caso de esta comunidad se replica en los pequeños oasis ubicados en las quebradas de la pampa alta situados al sureste de Arica, que forman parte de las cuencas hidrográficas de Codpa y de Camarones. Se pone énfasis en el papel que cumplen las relaciones sociales que los migrados mantienen con la localidad rural.

Para identificar las capacidades que posee el capital social de una localidad de precordillera en zonas hiperáridas y considerando los escenarios ambientales ya descritos se recurrió a la comunidad de Timar, constituida fundamentalmente por población de origen aymara, cuya principal actividad económica corresponde a la agricultura, la que desarrollan fundamentalmente en la quebrada de Carza o Calizama, que se encuentra en un sector de pampa alta próximo a la base del cordón occidental de la cordillera de los Andes (CIAT, 2011). En ese lugar se ubica el poblado homónimo a la comunidad, emplazado a unos 2.400 metros sobre el nivel del mar, área correspondiente a la comuna de Camarones, Región de Arica y Parinacota, que se encuentra a 90 kilómetros al sureste de Arica desde donde se accede por la carretera $\mathrm{CH}-5$ y la ruta A-31 (Imagen 1).

\section{Metodología}

El estudio se plantea desde un enfoque mixto de tipo descriptivo, con diseño no experimental y temporalidad longitudinal, para ello se recurrió a la aplicación de dos instrumentos, el primero consistente en una encuesta que indagó en el estado actual del capital social de la comunidad de Timar, mientras que el segundo consistió en la toma de 


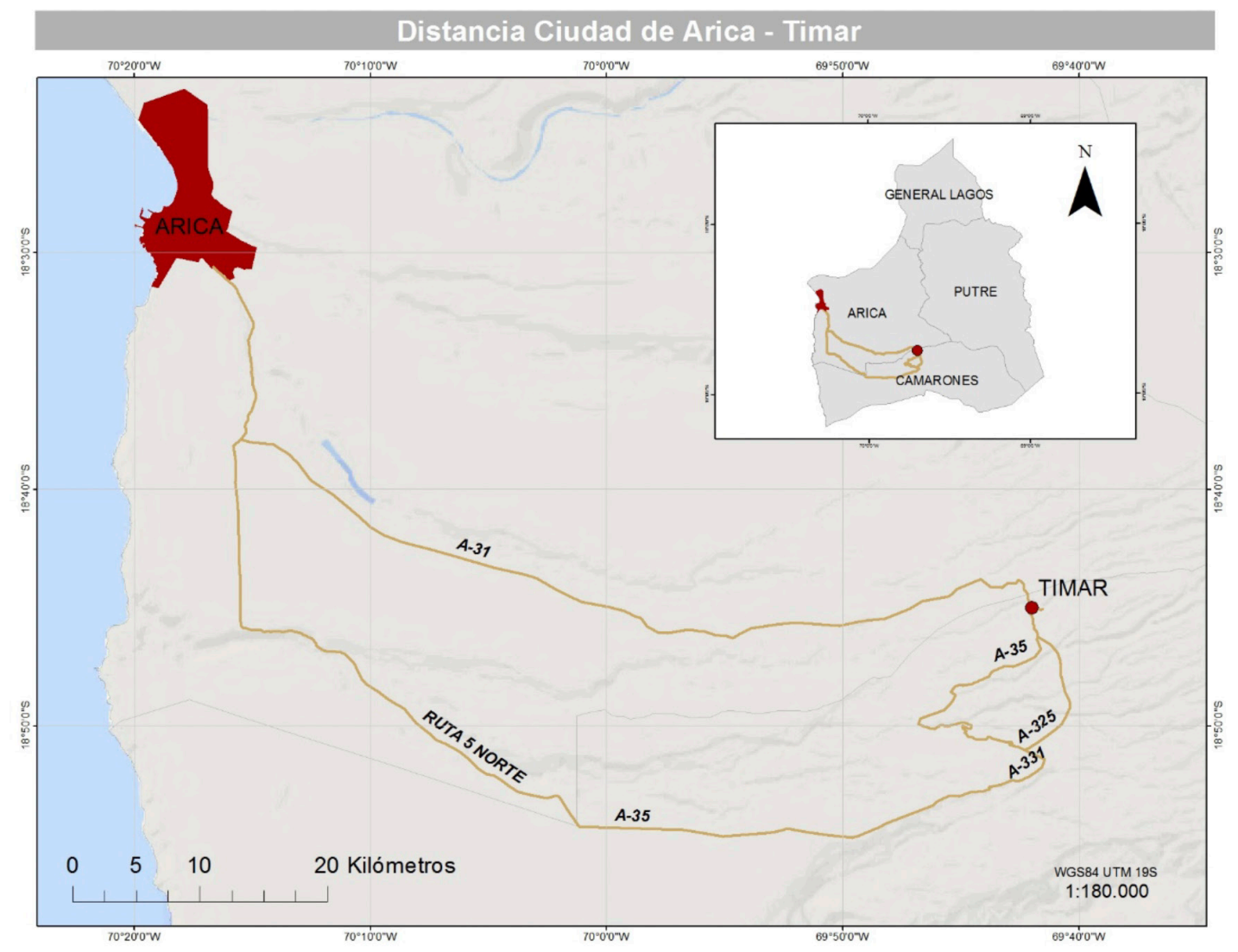

Imagen 1. Distancia entre la ciudad de Arica y la localidad de Timar. Fuente: Elaboración propia.

datos mediante entrevistas semiestructuradas a informantes claves para identificar las principales perturbaciones ambientales que afectan las actividades productivas desarrolladas en la quebrada.

La encuesta se conformó a partir de los ítems "Grupos y redes", "Confianza y solidaridad", "Acción colectiva y cooperación", "Cohesión social" y "Empoderamiento y acción política". Cada uno de ellos buscó poner de relieve aspectos fundamentales en los que se manifiesta el capital social en la quebrada de Timar, destacando entre ellos cuestiones como capacidad de injerencia en el desarrollo de las actividades comunitarias, unión entre miembros de la comunidad, nivel de participación y grados de solidaridad y asociatividad. Este instrumento se aplicó a trece personas con ocasión de la actividad comunitaria de limpieza de canales (faena) llevada a cabo tanto por residentes en la quebrada y miembros de la comunidad provenientes de la ciudad de Arica con los que los investigadores participaron activamente en la primavera del 2016.
Por su parte las entrevistas se aplicaron a diez informantes claves pertenecientes a la comunidad indígena de Timar que son residentes permanentes en el territorio de la comunidad. Este instrumento constó de tres ítems: "Actividades agrícolas", "Eventos climáticos extremos" y "Rol de las organizaciones sociales". El primer ítem centró sus preguntas en aspectos productivos y en la aplicación de un calendario agrícola, así como también en actividades económicas complementarias desarrolladas tradicionalmente. El segundo ítem consultó sobre perturbaciones ambientales de tipo local, el impacto de estas en el medio socionatural y la capacidad de ajuste y respuesta de la comunidad. Finalmente, el tercer ítem indagó en el rol de las organizaciones sociales existentes en el espacio de la comunidad y su capacidad para interrelacionarse con instituciones de carácter supralocal.

Esta metodología cualitativa junto con abordar los aspectos culturales que median la relación hombremedio y que en ocasiones no son visibilizados en 
enfoques cuantitativos (Adger et al. 2013), permite al investigador insertarse en las actividades de la comunidad para identificar las prácticas que dan cuenta de confianza y capital social (Withers, 2017). $\mathrm{Al}$ abordar aspectos relacionados con mecanismos de adaptación en un lugar específico como Timar, utilizando metodologías participativas, resultan particularmente propicias considerando que los efectos de las perturbaciones ambientales se desarrollan a nivel local al igual que lo hacen las respuestas de adaptación basadas en el capital social (Berkes y Jolly 2002)

\section{Resultados}

La movilidad no afecta el capital social adaptativo de la comunidad indígena de Timar, puesto que sus miembros, tanto residentes como migrantes, constituyen un recurso efectivo para responder a perturbaciones ambientales que desajustan el sistema socionatural de la comunidad. Entre las perturbaciones que tienden a alterar la estabilidad y el equilibrio del sistema socioambiental en Timar, los pobladores destacan los de orden natural asociados a fenómenos meteorológicos (lluvias, sequías, viento) y en menor medida geológicos (sismos) y biológicos (plagas) (Tabla 1).

\section{Lluvias}

En ocasiones durante el período estival originan grandes crecidas en los escurrimientos superficiales de la quebrada, pudiendo arrasar con canales y bocatomas, cuando ello se produce solo se reponen los daños a partir de marzo para evitar la recurrencia de la destrucción. Las lluvias, en el pasado, se presentaban en los sectores próximos al pueblo y hacia la quebrada, situación que no producía mayores daños, porque el agua bajaba en regueros desde los cerros sin producir daños mayores a las eras. Actualmente llueve más en las cabeceras de la cuenca de Timar (cerro Marques) originándose un mayor caudal entrante, lo que trae consigo la posibilidad de arrasar eras próximas al lecho de la quebrada o cortarlas. Cuando las lluvias exceden el umbral de ajuste del sistema socionatural es el capital social secundario y tercierio que se activa para responder a la disrrupción. En ese sentido se aplican medidas para mitigar los efectos adversos sobre las viviendas y áreas productivas.

\section{Sequía}

Aun cuando los agricultores destacan, en general, una disminución del recurso hídrico, señalan que tal fenómeno no les afecta en gran medida, debido a que el agua que utilizan para el regadío proviene de vertientes, las que presentan un caudal bastante estable. Para gestionar la escasez de agua en el caso de sequías, se realizan faenas comunitarias constantes que tienen como objetivo mantener limpio el canal, desmalezándolo y asegurando su nivel de pendiente para satisfacer las necesidades hídricas de las eras. Al mismo tiempo se aplica con

Tabla 1. "Perturbaciones ambientales y respuestas comunitarias".

\begin{tabular}{|c|c|c|c|}
\hline Evento & Resultado del evento & Forma de ajuste & Nivel de capital social \\
\hline Lluvias & $\begin{array}{l}\text { - Inundaciones. } \\
\text { - Activación de quebradillas } \\
\text { laterales. } \\
\text { - Corte de caminos y daños en } \\
\text { viviendas. } \\
\text { - Acumulación de barro en eras. }\end{array}$ & $\begin{array}{l}\text { - Realización de faenas extraor- } \\
\text { dinarias para la reparación de } \\
\text { canales y caminos. } \\
\text { - Protección de viviendas con } \\
\text { nylon. }\end{array}$ & $\begin{array}{l}\text { - Capital social secundario. } \\
\text { - Capital social primario. }\end{array}$ \\
\hline Sequía & - Deshidratación de frutales. & $\begin{array}{l}\text { - Limpieza y mantención de } \\
\text { canales de regadío. }\end{array}$ & - Capital social secundario. \\
\hline Viento & $\begin{array}{l}\text { - Pérdida de flores de árboles } \\
\text { frutales. }\end{array}$ & - No presenta. & - No presenta. \\
\hline Temblores & $\begin{array}{l}\text { - Derrumbes de material rocoso } \\
\text { sobre canales de regadío y } \\
\text { caminos. }\end{array}$ & $\begin{array}{l}\text { - Realización de faenas extraor- } \\
\text { dinarias para la reparación de } \\
\text { canales y caminos. }\end{array}$ & - Capital social secundario. \\
\hline Plagas & $\begin{array}{l}\text { - Merma en la producción } \\
\text { frutícola. }\end{array}$ & - Lavado de árboles. & - Capital social secundario. \\
\hline
\end{tabular}

Fuente: Elaboración propia. 
mayor rigurosidad el control y manejo del sistema de mitación del recurso.

\section{Viento}

La quebrada presenta vientos que perjudican algunos cultivos, en especial por la caída de las flores en los árboles frutales. En ocasiones se han destruido los techos en la localidad principalmente en noviembre. Para este caso no existen medidas mitigantes de tipo material, sino que se recurre a rogativas rituales asociadas a la cosmovisión andina que busca promover la armonía entre sociedad y naturaleza.

\section{Temblores}

Este tipo de fenómenos origina derrumbes de material rocoso desde las laderas de los cerros, material que obstruye los canales, interrumpe las vías de comunicación, inhabilitándolos. En estas ocasiones se realizan trabajos comunitarios para despejar canales y caminos con la finalidad de lograr abastecer de agua a los frutales evitando su deshidratación como también facilitar el intercambio de bienes y personas en la quebrada.

\section{Plagas}

Las variaciones en las condiciones térmicas e hídricas en el territorio de la comunidad de Timar en ocasiones propician la aparición de pestes que afectan fundamentalmente a los árboles frutales del lugar, trayendo consigo merma en la producción y consecuentemente en los ingresos de los comuneros. Para estos casos la comunidad cuenta con una bomba de agua para lavar los árboles y retirar el material infeccioso de las hojas y las flores, de igual forma, los testimonios indican el trabajo comunitario para ir predio por predio limpiando y en ocasiones fumigandos los árboles.

Frente a situaciones especiales, como aquellas asociadas a perturbaciones de índole natural, que constituyen alteraciones en el funcionamiento normal del sistema socionatural de la comunidad, se hace presente el sentido de unión de los comuneros que los lleva a acordar medidas para la ejecución de trabajos conjuntos para alcanzar fines comunes, así como se produce la activación de redes de cooperación que se constituyen en la base para la acción colectiva. Un papel importante reviste en estas ocasiones la común pertenencia y adscripción cultural (aymara) de los comuneros como factor de cohesión social y de aprendizaje colectivo debido a la capacidad para aportar respuestas creativas e imaginativas con las cuales solucionar problemas y disfunciones e impulsar medidas de adaptación y de fortalecimiento de la capacidad de resiliencia ante disrupciones ligadas a cambios ambientales.

El cambio que significó para Timar y la comunidad del lugar los procesos de migración y movilidad además de generar una importante disminución de la población asentada en el poblado, también afectó al sector de Cobija, localidad ubicada más al interior, donde gran parte de agricultores timareños disponían también de tierras de cultivos y practicaban el modelo andino de control de pisos ecológicos, lo que les permitía obtener distintos productos agrícolas: frutas en Timar, y papas, orégano y alfalfa, así como preparación de chuño en Cobija. La explotación de los diferentes pisos altitudinales prácticamente desapareció por la disminución de la fuerza de trabajo, por el cambio de lógica económica orientada hacia una de mercado y el envejecimiento de los agricultores del sector; a lo que se suma la disminución de animales de carga como resultado de la falta de pastos forrajeros, los que dejaron de cultivarse cuando comienza el proceso de migración, situación que limitó el traslado de la producción desde los predios de difícil acceso, trayendo como consecuencia la merma en el ingreso familiar.

Así queda evidenciado por medio de los testimonios entregados por los comuneros quienes se refieren a los cambios ocurridos. Antiguamente, los pobladores de Timar se movilizaban por temporada en diferentes sectores del territorio de la comunidad; en el pueblo permanecían durante la temporada de poda y cosecha de la fruta; a Cobija se dirigían durante la temporada de papa y maíz y para la preparación de chuño; a la quebrada de Apanza acudían cuando las aguas lluvias bajaban por unos meses, con la finalidad de aprovechar esos recursos mediante cultivos estacionales (Pereira 2015). En ocasiones se desplazaban a trabajar en la cosecha de aceituna en el valle de Azapa y para la fiesta religiosa de "Virgen de las Peñas" se hacían fletes y se aprovechaba la ocasión para vender y comprar animales permitiendo su renovación debido a la concurrencia de arrieros de diferentes pueblos.

Entre diciembre y marzo se comerciaba la producción frutícola en Arica, productos trasladados a 
lomo de burros y mulas, regresándose con víveres requeridos durante el año, como harina, arroz, trigo, charqui, entre otros. A su vez, pobladores del altiplano acudían en caravanas hasta la quebrada a realizar trueque de carne de llamo por fruta fresca y deshidratada.

Empero los cambios suscitados, los resultados de la investigación dan cuenta que las redes sociales de migrantes, en el caso de Timar, han ayudado al capital social en esta comunidad de origen, aumentando la resiliencia y la capacidad de adaptación ante perturbaciones presentes en el medio, pudiéndose identificar vinculaciones que presentan este tipo de personas con la comunidad del lugar y su disposición a colaborar con ella ante perturbaciones que puedan afectarla.

La población timareña migrada y asentada en la ciudad de Arica ha seguido manteniendo vínculos con su lugar de origen, no solo favorecidos por la proximidad social y cultural existente entre las personas, sino también por las de índole espacial como la distancia física. Estas condiciones, que ayudan a la conformación de redes de cooperación, si bien no poseen una expresión permanente, tienden a activarse en ocasiones especiales, como ante la ocurrencia de disrupciones que afectan el normal funcionamiento del sistema socionatural.

De las respuestas entregadas por las personas encuestadas destaca la existencia de un marcado interés por involucrarse en labores de cooperación con los residentes de la comunidad en la quebrada, en especial cuando se presentan perturbaciones asociados a fenómenos naturales. Dichas perturbaciones tienden a afectar al grupo social en su conjunto originando alteraciones en el ámbito productivo y en la conservación del patrimonio cultural tradicional; la disposición a prestar apoyo constituye una expresión del sentido de pertenencia por parte de los migrantes hacia la comunidad de Timar.

Ante consultas relacionadas con medidas de apoyo y contribuciones que se podrían llevar a cabo hacia la comunidad en Timar, la mayoría de los encuestados se muestra dispuesto a colaborar con tiempo y dinero (Gráfico 1). De hecho, alrededor del $70 \%$ de ellos participa en las faenas comunitarias (Gráfico 2). A lo largo del tiempo han mantenido sus redes de colaboración y en algunos casos estas han experimentado un aumento, lo que tiene relación con el fortalecimiento de los lazos de confianza existentes (Gráfico 3).
A pesar de las adversidades, la capacidad de trabajo conjunto en Timar permanece vigente, destacándose acciones de beneficio mutuo que se practican en los trabajos comunitarios realizados en las labores denominadas "Faenas", estas son jornadas de trabajo agrícola que llevan a cabo todos los comuneros un fin de semana determinado, con la finalidad de proceder a la limpieza de las vertientes y de los canales, eliminando malezas y evitando con ello pérdidas de agua para el regadío de los frutales. Las normas que rigen la faena obligan a la participación de todos los comuneros que poseen chacra en la quebrada, aplicándose multas para aquellos que no asisten. Con ocasión de la celebración de la fiesta de San Juan se efectúa una faena con la finalidad de limpiar y preparar la iglesia y el pueblo para recibir a los miembros de la comunidad y asistentes a la fiesta patronal.

Los esfuerzos de los trabajos comunitarios son dedicados al control y manejo de los recursos hídricos que se disponen en la quebrada, los que se obtienen de los aportes entregados por seis vertientes que se presentan en el área, cinco de ellas ubicadas en las proximidades de Timar y una en la quebrada aguas abajo del pueblo. Se utiliza la mita como institución tradicional de repartición por turno del agua para el riego, distribuyéndose el agua según la superficie de terreno que posee el regante. A partir de los estanques de acumulación se transfieren volúmenes que van de medio estanque a estanque completo. Las aguas provenientes de las vertientes, tradicionalmente consideradas comunitarias, fueron inscritas a nombre de la Comunidad Indígena de Timar oficialmente el 2005, como forma de asegurar la propiedad y evitar la intromisión de otros intereses que puedan disputar tales recursos, como ha sucedido en otras comunidades del norte del país.

La disminución de la población dedicada al trabajo agrícola y la menor disponibilidad de agua, declarada por los encuestados, ha traído consigo una modificación en el antiguo patrón de cultivos existente en Timar, es así que cultivos tradicionales como maíz, papas y hortalizas, que requerían riego cada ocho a diez días, han dejado de practicarse, de manera que en la actualidad algunas hortalizas solo se siembran con la finalidad de autoconsumo, la dedicación fundamental en la actualidad se centra en los árboles frutales, cuyo riego se realiza cada veinticinco a treinta días. 


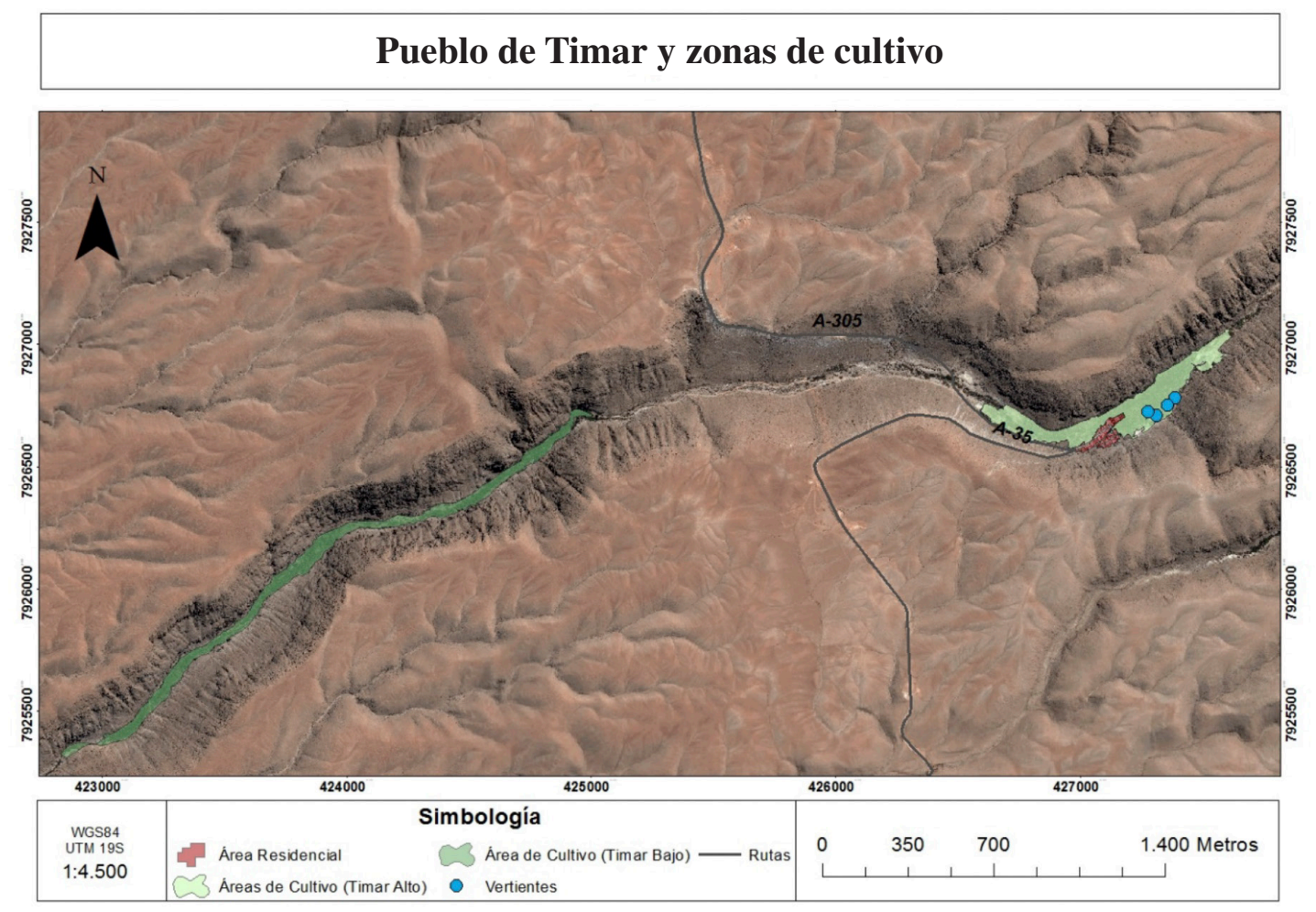

Imagen 2. Pueblo de Timar y Zonas de Cultivo. Fuente: Elaboración propia.

\section{¿Cómo contribuye a la comunidad local la permanencia de su vínculo?}

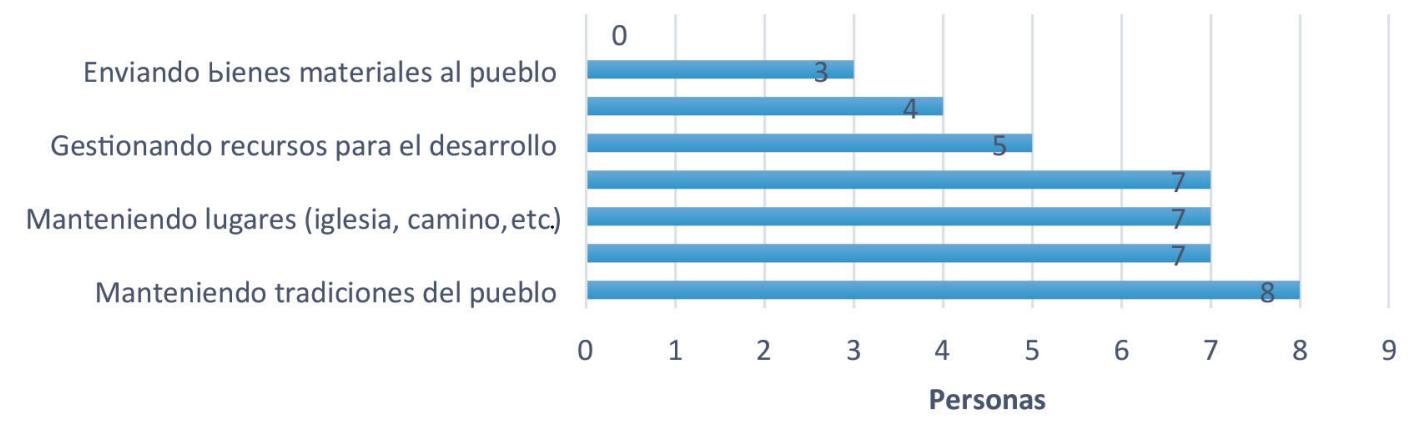

Gráfico 1. Tipos de contribución a la permanencia del vínculo de la comunidad local. Fuente: Elaboración propia.

Respecto de la condición residencial de los comuneros de Timar destaca el hecho del autorreconocimiento de tres categorías de adscripción espacial. Primeramente, los migrados asentados en la ciudad de Arica que se desempeñan en trabajos que no presentan relación con la agricultura, mantienen vinculaciones con la comunidad de origen, en especial lazos que nacen de la condición familiar que poseen con comuneros a los que visitan en contadas ocasiones en Timar, en especial en días feriados, oportunidades en que prestan colaboración, aunque no permanentemente, en el ámbito productivo. $\mathrm{La}$ mayoría asiste al pueblo por razones de índole religiosa, con la finalidad de celebrar las fiestas 


\section{Durante el año pasado ¿participó en las faenas comunitarias en Timar?}

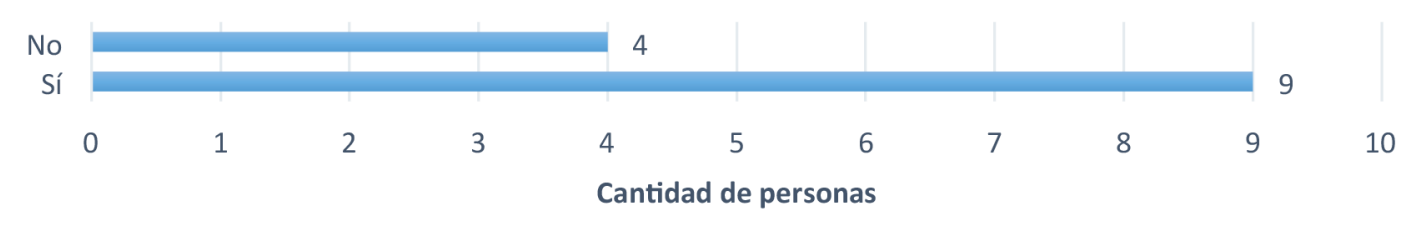

Gráfico 2. Grado de participación en faenas comunitarias. Fuente: Elaboración propia.

\section{¿Cómo han variado en los años sus redes sociales en Timar?}

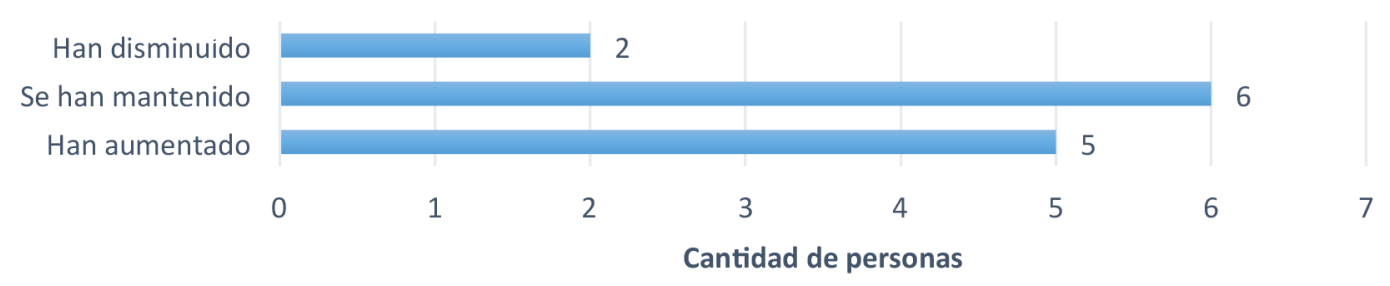

Gráfico 3. Variación de las redes sociales en Timar. Fuente: Elaboración propia.

tradicionales, entre ellas la del santo patrono del pueblo, San Juan.

En la segunda categoría de personas vinculadas a Timar es posible identificar residentes rurales o habitantes permanentes del pueblo. Son básicamente fruticultores de especies cítricas (naranjas, limones, limas), además de otros productos como peras, ciruelas, duraznos, manzanas, tumbos y paltas, producción que está dirigida a ser comercializada en el mercado regional. Cultivos de hortalizas como papas, maíz (choclo), habas, arvejas, entre otros, solo son realizados con fines de autoconsumo. Las áreas productivas se presentan en el fondo de la angosta quebrada y están conformadas por pequeñas propiedades fraccionadas que corresponden a chacras de alrededor de $2.000 \mathrm{~m}^{2}$, que se abastecen de aguas de vertiente para el regadío, afloramientos permanentes en la quebrada cuyos aportes provienen del escurrimiento subterráneo de las lluvias estivales y deshielos que ocurren en la alta cordillera (Imagen 2).

Finalmente, en una tercera categoría se encuentran quienes declaran tener una doble residencia; son sujetos vinculados al territorio, principalmente por haber nacido ahí o por poseer vínculos familiares vigentes con los moradores permanentes, esta doble residencia no implica predominancia de una sobre la otra. Alternan su estadía entre la ciudad y el pueblo, algunos de ellos poseen, además de vínculos familiares, terrenos productivos que complementan sus ingresos; participan de la actividad comunitaria de la localidad, sin embargo no se consideran completamente habitantes rurales.

La interrelación que a lo largo del tiempo ha mantenido la comunidad timareña con el entorno de la quebrada le ha permitido desarrollar un alto nivel de integración con el medio natural, construyendo su territorio, y a partir del conocimiento alcanzado manejar los recursos disponibles y al mismo tiempo desplegar medidas de adaptación al mismo. En tal sentido destaca el uso y manejo de los tiempos del calendario agrícola del lugar (Tabla 2).

La metodología utilizada permitió constatar la existencia de capital social adaptativo, que se sustenta en el actuar colectivo reforzado por los lazos culturales, la identidad, la cohesión y el sentido de lugar de los miembros de la comunidad, independiente del área de residencia. 
Tabla 2. Calendario agrícola en Timar.

\begin{tabular}{cc}
\hline Actividad & Meses \\
\hline Cosecha de verano & Diciembre - enero - febrero - marzo \\
Poda, limpieza, fertilización orgánica & Mayo y junio \\
Brotes y floración & Agosto \\
Cuidado de árboles, caída de flores por viento & Septiembre \\
Tiempo de siembra & Agosto y septiembre \\
Último período de siembra & Octubre \\
Cosecha (lo sembrado en agosto-septiembre) & Enero \\
Cosecha (de siembras tardías) & Abril - mayo \\
\hline
\end{tabular}

Fuente: Elaboración propia.

\section{Conclusiones}

El capital social de la comunidad de Timar posee una base rural sustentada en los integrantes de las organizaciones ubicadas en la quebrada, compuestos en su mayoría por personas de edad avanzada que se desempeñan en labores agrícolas; y una base urbana mantenida en los migrantes asentados en Arica que no se han desvinculado de la comunidad del lugar y participan eventualmente en actividades comunitarias.

En coincidencia con lo planteado por Scheffran, et al. 2012, los individuos migrados que no se han desvinculado completamente de la comunidad constituyen una parte importante del capital social local, facilitando la formación de redes para la localidad de origen.

Acorde a lo señalado por Glaeser et al. 2002, en el área de estudio las conexiones sociales, base del capital social estructural, no han decaído producto de la distancia física y de los costes de viaje, sino por el contrario, se han mantenido considerando la proximidad física y conectividad entre la ciudad y el pueblo, a diferencia de lo que se ha podido constatar en otras áreas de la región distante de la ciudad como la localidad de Caquena ubicada en el altiplano (López et. al 2017) .Un papel importante reviste en estas ocasiones la común pertenencia y adscripción cultural (aymara) de los comuneros como factor de cohesión social y de aprendizaje colectivo, a causa de la capacidad para aportar respuestas creativas e imaginativas con las cuales solucionar problemas y disfunciones e impulsar medidas de adaptación $\mathrm{y}$ de fortalecimiento de la capacidad de resiliencia ante disrupciones ligadas a cambios ambientales (Caravaca y González, 2009).

La relación de los emigrados con la comunidad del lugar no solo está mediada por el interés económico de participar de los beneficios obtenidos de la producción de frutas y cítricos, sino también por un afán de preservar las tradiciones y contribuir a la conservación histórica del pueblo mediante la mantención de prácticas comunitarias, religiosas y simbólicas del lugar.

Los lazos sociales dan cuenta de la existencia de un capital social, tanto localizado como translocalizado, que constituye un recurso que permite dar respuesta a perturbaciones ambientales y que constituyen expresión de territorialidad, en la medida que sus miembros mantienen su sentido de pertenencia a la comunidad local.

El proceso de emigración que afectó a pueblos y comunidades como Timar significó un cambio sustantivo en el modelo tradicional de ocupación y asentamiento en el territorio de la comunidad tradicional, si bien se tradujo en una disminución de la población asentada permanentemente en el lugar, dio paso a una forma distinta de habitabilidad y territorialidad. El desplazamiento de población a la ciudad no implicó un total abandono de prácticas sociales que hacían posible la conformación de redes que vinculaban a Timar con diversos pisos ecológicos distintos al de la ciudad y con otras comunidades rurales, cuestión que significó el fortalecimiento de nuevas redes para la comunidad vinculadas con el centro urbano y los valles costeros de la región.

Aun cuando el capital social de Timar se vio afectado por el cambio experimentado en la comunidad, muchas prácticas persisten en su seno guiadas por una regulación a base de normas, sanciones y tradiciones, lo que ha permitido detentar una comunidad resistente, con pertenencia e identidad territorial; la posibilidad de acceder a un capital social translocalizado, le permite disponer de un recurso al que acude ante situaciones que signifiquen 
alteraciones graves en su accionar como sistema, ya que la gente emigrada posee un vínculo real, potente y comprometido con la localidad.

El estado actual del capital social de Timar es un recurso para la adaptación, porque su comunidad, a pesar de estar dispersa en el espacio de la región, posee un vínculo permanente con la quebrada.

\section{Agradecimientos}

Los autores agradecen al proyecto FONDECYT 1150701 , al proyecto de Investigación de la Universidad de Tarapacá, UTA MAYOR 5759/17, al Convenio de Desempeño UTA-MINEDUC y muy especialmente a los hombres y mujeres de la comunidad indígena de Timar.

\section{Referencias Citadas}

Aceituno, P.

1997 Elementos del Clima en el Altiplano Sudamericano. Revista Geofisica (44). Pp. 37-55

Adger, W. N., Barnett, J. Brown, K. Marshall N. y O’Brien, K. 2013 Cultural dimensions of climate change impacts and adaptation. Nature Climate Change 3, Pp. 112-117.

Adger, W. N.

2003 Social Capital, Collective Action, and Adaptation to Climate Change. Economic Geography, Pp. 387-404.

Adger, W. N.

2006 Vulnerability. Global Environmental Change 16 pp. 268-281

Adger, W. N., Arnell, N. W., y Tompkins, E. L.

2005 Successful adaptation to climate change across scales. Global Environmental Change, 15(2), Pp, 77-86.

Alvarado, L.

1970 La vida rural en el Altiplano Chileno. Santiago: ICIRA, 1970.

Arriagada I.

2003 Capital social: potencialidades y limitaciones analíticas de un concepto. Estudios sociológicos (21). Pp. 557-584. Disponible en: http://www.jstor.org/stable/40420794

Berkes, F., Jolly, D.

2002 Adapting to Climate Change: Social-Ecological Resilience in a Canadian Western Arctic Community. Ecology and Society 5.(2). Disponible en: http://www.consecol.org/ vol5/iss $2 /$ art 18

Caravaca, I. y González, G.

2009 Las redes de colaboración como base del desarrollo territorial. Scripta Nova. Revista Electrónica de Geografía y Ciencias sociales. Barcelona: Universidad de Barcelona, 1 de mayo de 2009, vol. XIII, núm. 289 http://www.ub.es/ geocrit/sn/sn-289.htm. [ISSN: 1138-9788].

Comunidad Indígena Aymara de Timar [CIAT]

2011 Timar: Historia y costumbres de un pueblo andino en el norte de Chile. Corporación Nacional de Desarrollo Indígena, Ministerio de Desarrollo Social, Gobierno de Chile.

Foronda-Robles, C., y Galindo-Pérez de Azpillaga, L. 2012 Argumentación relativa a la confianza territorial. Claves sobre capital social. Cuadernos de Desarrollo Rural, 41-63.

Glaeser, E., Laibson, D. y Sacerdote, B.

2002 An Economic Approach to Social Capital. The Economic Journal, 112, Pp. 437-458.

Gundermann, H., y Vergara, J.

2009 Comunidad, organización y complejidad social andinas en el norte de Chile. Estudios Atacameños. (38). Pp. 107-126.
González, H.

1990. Timar, diagnóstico socioeconómico. Acerca de la utilización de dos espacios económicos: el campo y la ciudad. Serie Documentos de Trabajo.

IPCC.

2013 Climate Change 2013: The Physical Science Basis, Fifth Assessment Report of the Intergovernmental Panel on Climate Change. Cambridge University Press: Cambridge, UK. http:// http://ipcc.ch/report/ar5/wg1/

López-Cepeda, J. F., Tapia, A. y Romero, H.

2017 Capital Social y Respuestas a Perturbaciones Ambientales en la Comunidad Andina de Caquena, Norte de Chile. Interciencia. Vol. $42 \mathrm{~N}^{\circ}$ 7. Pp. 430-436.

Mendonça, $\mathrm{M}$.

2013 Monzón Sudamericano: La integración de la Circulación Amazónica y Altiplánica y las Variabilidades Climáticas del Altiplano Andino Chileno. XIV Encuentro de Geógrafos de América Latina (EGAL Lima 2013). http://observatoriogeograficoamericalatina.org.mx/egal14/Procesosambientales/ Climatologia/28.pdf

Meseguer-Ruiz, O., Corvacho, O., Tapia, A., López, J.F. y Sarricolea, P.

2016 Tendencias de la Temperatura Mensual y de los Extremos Diarios durante el Período 1966-2015 en el Norte Grande Chileno. X Congreso Internacional AEC: Clima, sociedad, riesgos y ordenación del territorio DOI: http:// dx.doi.org/10.14198/XCongresoAECAlicante2016-24

Núñez, L.

1974 La agricultura prehistórica en los Andes meridionales. Editorial Orbe.

Padigala, B.

201 Social Capital and Local Institutions: A Perspective to Assass Communities Adaptation Potential to Climate Change, en Filho, W.L. (ed.): Handbook of Climate Change Adaptation, Springer Berlin Heidelber, pp. 1927.1950

Pereira, K.

2015 Adaptación a la variabilidad climática. Caso de los cultivos estacionales en la Quebrada de Apanza, Cuenca de Codpa, Región de Arica y Parinacota. Memoria de Título Geógrafo. Universidad de Tarapacá.

Romero, H., Smith, P., Mendonça, M., y Méndez, M.

2013 Macro y mesoclimas del altiplano andino y desierto de Atacama: desafíos y estrategias de adaptación social ante su variabilidad. Revista de Geografía Norte Grande, (55), 19-41. https://dx.doi.org/10.4067/S0718-34022013000200003

Sarricolea, P. y Romero, H.

2015 Variabilidad y cambios climáticos observados y esperados en el Altiplano del Norte de Chile. Revista de Geografía 
Norte Grande No. 62 Santiago. http://dx.doi.org/10.4067/ S0718-34022015000300010

Scheffran, J., Marmer, E., y Sow, P.

2012 Migration as a contribution to resilience and innovation in climate adaptation: Social networks and co-development in Northwest Africa. Applied Geography, 33. Pp. 119-127.

\section{Nota}

1 En este punto es importante recordar que las zonas rurales del norte de Chile poseen baja población. De hecho, el

\section{Withers, C.W}

2017 Trust -in Geography. Progress in Human Geography. Disponible en: http://journals.sagepub.com/doi/ full/10.1177/0309132516688078

censo del 2002 señala que la población del pueblo de Timar alcanzaba a 17 habitantes. 